\title{
Nueva localidad para Senecio gamolepis Cabrera (Asteraceae) en los Altos Andes del Sur peruano
}

\section{A new locality for Senecio gamolepis Cabrera (Asteraceae) in the High Andes of Southern Peru}

\begin{abstract}
Alfredo Tupayachi Herrera
Profesor Investigador Asociado al Herbario (CUZ), Universidad Nacional de San Antonio Abad del Cusco. Av.de la Cultura, 733 Cusco,Perú.atupayachi@yahoo.com

\section{Resumen}

Durante el desarrollo del Proyecto GLORIA, entre los años 2002 y 2012, se logró reportar una nueva localidad de Senecio gamolepis Cabrera. Esta nueva localidad es para los altos andes de la Cordillera de Vilcanota, al Norte de la meseta de Sibinacocha y próximo a los complejos nevados en la Región Cusco. La nueva localidad comprende altitudes de 4600 a 5370 m; donde la especie forma cojines, es leñosa y de flores amarillas, vegetando sobre suelos arenosos y entre rocas de las formaciones morrénicas, producto de la desglaciación. Esta investigación permite ampliar el conocimiento acerca de su distribución hasta la fecha y por tanto tiene interés Fitogeográfico.
\end{abstract}

Palabras Clave. Cordillera deVilcanota, nueva localidad, Senecio gamolepis.

\begin{abstract}
During the development of the GLORIA Project, between 2002 and 2012, it was possible to report a new locality of Senecio gamolepis Cabrera, for the high Andes of the Vilcanota Mountain Range, north of the Sibinacocha plateau and close to the snowy mountain range in the Cusco Region; the new localities cover altitudes of 4600 to $5370 \mathrm{~m}$; where the species forms cushions, they are woody and they have yellow flowers. They are vegetating on sandy soils and among rocks of morrenic formations, product of deglaciation. This research allows us to expand our knowledge about its distribution to date, and therefore has Phytogeographic interest.

Key words. new locality, Senecio gamolepis, Vilcanota Mountain Range.

\section{Introducción}

El Perú, como uno de los integrantes de la Comunidad Andina fue seleccionado junto a Colombia, Ecuador, Bolivia y Argentina, por el Proyecto Global Observation Reseach Initiative in Alpine Enveronment "GLORIA" (Iniciativa para la Investigación y el Seguimiento Global de los Ambientes Alpinos),

cuyo objetivo principal es el de establecer una red de observaciones a largo plazo que permitan desarrollar estudios comparativos de los impactos del cambio climático en la biodiversidad de alta montaña y desarrollar un protocolo de seguimiento estándar en las principales cordilleras de la tierra, centrando su atención en áreas de alta montaña, únicas unidades biogeográficas distribuidas en todo el-
\end{abstract}


-en todo el mundo (Komer, 1999).

Para cumplir con objetivos del proyecto, se eligió a la Cordillera de Vilcanota en Canchis Cusco, como el primer sitio GLORIA en Latino América, para realizar estudios interconectados impulsados por el cambio climático sobre la biodiversidad organizándose la expedición científica a la Cordillera de Vilcanota, en este marco del proyecto se instaló la primera de las cuatro cimas del sitio Gloria en Cusco - Canchis, Cordillera de Vilcanota denominado "Riti T'ica” (en el año 2003). En cuya ocasión, en las parcelas de observación permanente y la cobertura de la vegetación circundante en base al protocolo estandarizado por la iniciativa para la investigación, entre otras especies se colectó por primera vez a Senecio "cousin” sp nova?, al estado estéril por Halloy et al, s/n. 15 agosto 2003 y entregado al Herbario (CUZ).

En 2005, como integrante de la expedición a la Cordillera de Vilcanota colecté muestras en floración y se determinó como Senecio gamolepis Cabrera confirmado por Hamilton Beltrán curador del Museo de Historia Natural de la Universidad Nacional Mayor de San Marcos (2006); desde entonces durante expediciones sucesivas en los años 2008, 2011 y 2014 se volvió a encontrar a la especie formando cojines en espacios donde la vegetación no sufre directamente los impactos humanos o lo hace de modo menos intenso que a altitudes inferiores; desde entonces consideramos a la alta montaña de la Cordillera de Vilcanota al Noreste de la Laguna de Sibinacocha en Pitumarca, Canchis- Cusco, como nueva localidad para esta especie en el Sur del Perú, con lo cual se amplía su distribución.
La familia Asteraceae comprende alrededor de 250 géneros y 1590 especies (Brako \& Zarucchi, 1993; Ulloa Ulloa et al., 2004); mayormente están formados por hierbas, arbustos y subarbustos; los géneros con mayor número de especies son: Senecio, Gynoxys y Verbesina . Senecio con 93 especies endémicas para el Perú (Beltran et al. 2006); Senecio gamolepis había sido colectada sólo en los departamentos de Junín y Lima en los Andes III entre 4000 a 4500 m. (Gentry, A., 2003) Reseña de la Flora Peruana XXIX-XXXVIII. (En Brako \& Zarucchi, 1993). Hasta el año 2003 se había registrado solo en 4 departamentos: Ancash, Huancavelica, Junín y Lima entre 4000 y 4500 m. (Beltran, 2016).

Senecio canoi y S. sykorae son consideradas morfológicamente similares a $S$. gamolepis (Montesinos et al. 2015), quienes manifiestan que el carácter más visible para diferenciar las tres especies son las hojas, que en $S$. canoi son oblongo - espatuladas y dentadas; $S$. sykorae obovado - espatuladas, mientras que en $S$. gamolepis son enteras y linear- lanceoladas.

\section{Material y Métodos}

\section{Área de estudio.}

El estudio se realizó al Noreste de la Laguna Sibinacocha en Inmediaciones de los nevados Jatun Rit'i y Oskollo Hananta de la Cordillera deVilcanota entre altitudes de 4890 a $5370 \mathrm{~m}$, a los $\left(13^{\circ} 46^{\prime} \mathrm{S}, 71^{\circ} 05 \mathrm{~W}\right)$, Cuenca alta de la Laguna de Sibinacocha, territorios de alta montaña pertenecientes al distrito de Pitumarca provincia de Canchis de la Región Cusco. (Fig. 1). 

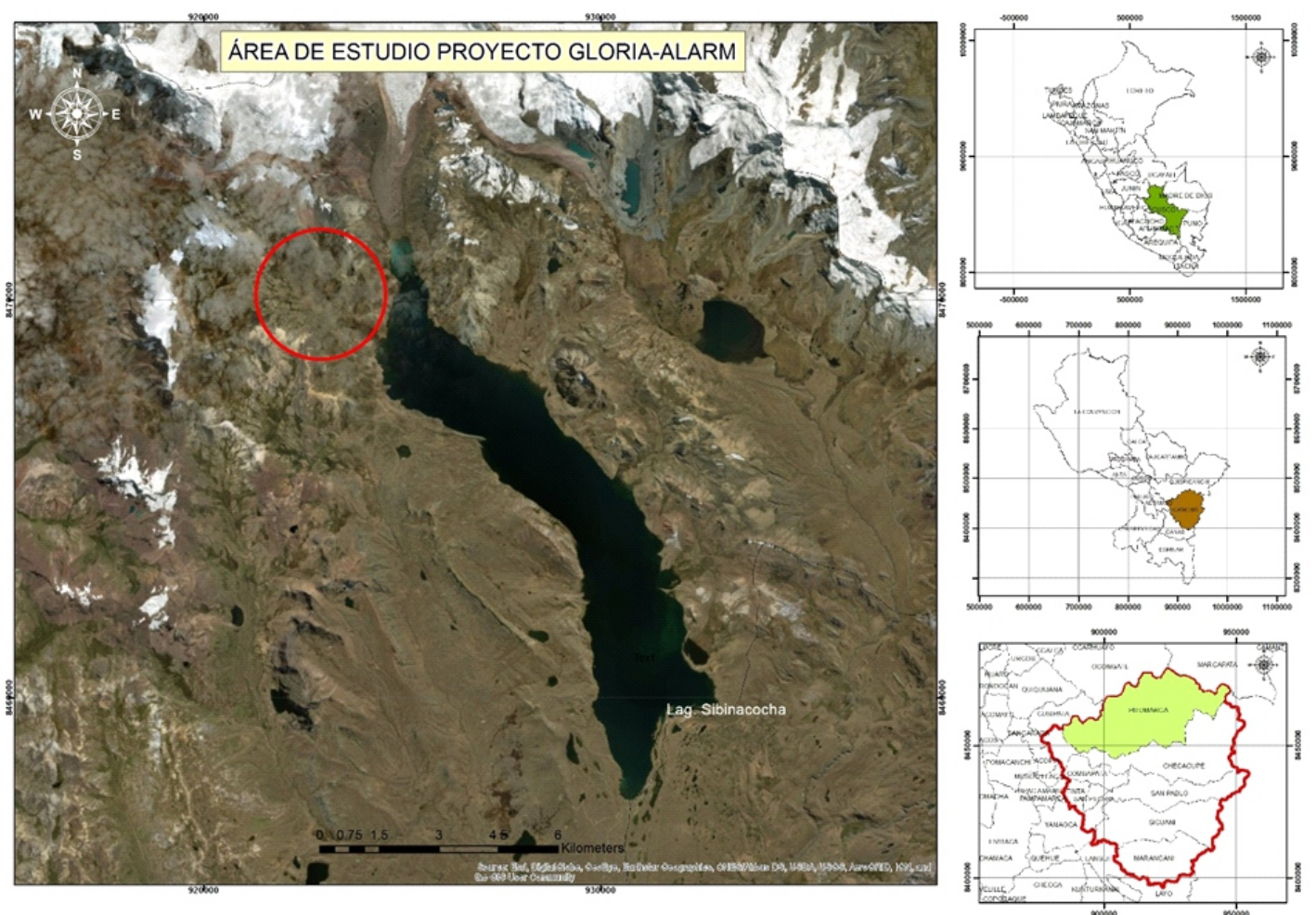

Figura 1. Área de estudio: Norte de la Laguna Sibinacocha-Cusco- Perú (SIB-PE). 2017.

\section{Metodología}

El estudio corresponde a los o a los objetivos del Proyecto GLORIA de cuantificar los cambios en los patrones de diversidad y distribución de las especies y/o comunidades vegetales respecto a los factores ambientales siguiendo la gradiente altitudinal, para comparar el modelo de diversidad desarrollado de manera extensiva en Europa con datos obtenidos en países seleccionados y sitios cimeros instalados en Sudamérica, con el establecimiento de nuevas especies después de recientes pérdidas de los glaciares (Manual para el trabajo de campo del Proyecto Gloria,2003). Las cuatro cumbres evaluadas del Sitio Gloria en la Cordillera deVilcanota y en orden cronológico de instalación son:
Rit'iT'ica a los 5250 m ( 2003), Pumachunta a 4890 m (2008), Orqo Q'ocha a 5325 (2008) y Yurak a 5498 m. (2012) (Informe del Trabajo de campo en la Cordillera de Vilcanota SIBPE,2008) En cuyas parcelas permanentes y flora circundante de las montañas donde destacan vegetación rala de gramíneas asociadas a hierbas bajas de los géneros Senecio, Xenophyllum, Werneria, Valeriana, Nototriche, Pycnophyllum, Calamagrostis, Baccharis, y Mniodes así como el césped de la puna formada por especies almohadilladas y formando placas, siendo una de éstas Senecio gamolepis.

Las colecciones se realizaron desde el 2003 Halloy et al s,n; Tupayachi et al. $N^{\circ} .6404$ (2005). 
- Tupayachi et al, $\mathrm{N}^{\circ} .8218$ (2011); Tupayachi et al, $\mathrm{N}^{\circ} .8285$ (2011); Tupayachi et al, $\mathrm{N}^{\circ} .8492$ (2014), cuyas muestras se encuentran depositadas en el Herbario (CUZ) de la Universidad Nacional de San Antonio Abab del Cusco. Complementariamente se recurrió a consultar en Trópicos (http: www.tropicos.org) donde aparece $\underline{S}$. gamolepis en dos departamentos: Ancash, Huaráz: Pasto Ruri; Parque Nacional de Huascarán en la Cordillera Blanca, colectado por L.W. Peterson s.n (MO), 4500 m, de 1988 y otro en Lima, Canta Para el Césped de la Puna inmediaciones del Nevado La Viuda, colectada por Irene Meza 213 (MO) a 4250 m, 1963. De igual forma se consultó a Brako \& Zarucchi, 1993, donde S. gamolepis está distribuido en los departamentos de Junín y Lima, considerada como hierba endémica para los Andes III 4000$4500 \mathrm{~m}$, cuyo Voucher es de Asplund 11820 (S).

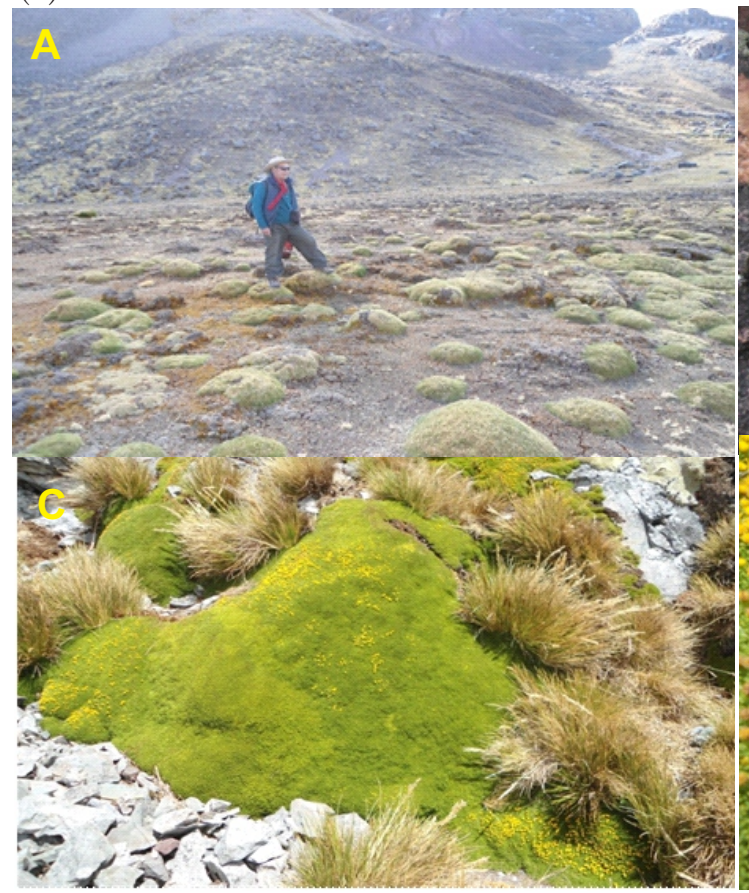

Figura 2. A. Deyeuxia chrysophylla Phil. Formando cojines, B. Senecio gamolepis Cabrera, Rodeada de Deyeuxia nitidula (Pilg.), C. Mniodes sp. "Laka laka” Placas sobre rocas y D. S. gamolepis Cabrera, formando cojines sobre

\section{Resultados}

De la evaluación en las cumbres cimeras de la flora de alta montaña en el marco del Proyecto GLORIA, se desprende que las altas montañas de la Cordillera de Vilcanota corresponden a ambientes poco explorados florísticamente no sólo a falta de financiamiento económico sino básicamente por factores climáticos extremos y altitudes que sobrepasan los $5000 \mathrm{~m}$, que no permiten realizar trabajos sostenidos especialmente cuando corresponden a la antesis floral coincidentes con la estación lluviosa: sin embargo, durante las exploraciones, en la estación de secas, en las cumbres cimeras del proyecto Gloria, áreas aledañas y otras localidades de la cuenca alta de Sibinacocha se ha encontrado a Senecio gamolepis en floración formando cojines libres o asociados a otras especies, tanto en suelos arenosos y rocosos-

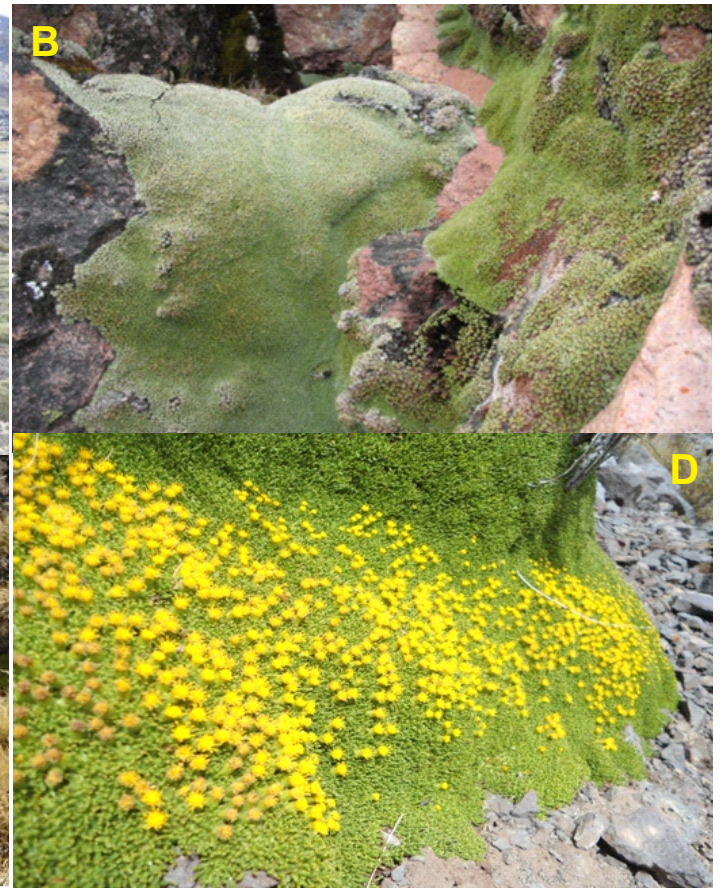




\section{Ecología}

En la Región Cusco, se distribuye entre altitudes de 4600 a $5370 \mathrm{~m}$, al Noreste de la Cuenca alta de la Laguna de Sibinacocha en el ámbito de alta montaña perteneciente al distrito de Pitumarca, provincia de CanchisCusco, formando cojines cespitosos del biotipo: Caméfitos pulvinados (Raunkiaer,1934), en esta gradiente prefiere suelos arenosos y pedregosos abiertos en laderas morrénicas y ambientes de reciente retroceso glaciar; su período de floración en la zona es entre marzo a agosto.

Estado de conservación: VU B1ab(iii) - Vulnerable - Global (León et al. 2006; IUCN 2001).

\section{Discusión}

Los ejemplares colectados por Peterson en la Cordillera Blanca en Huaráz y por Meza en la Cordillera La Viuda en Canta, (4500 - 4250 $\mathrm{m})$, están por debajo de la altitud a la que encontramos en la Cordillera de Vilcanota (5000 - $5370 \mathrm{~m})$. Igualmente, en referencia al biotipo Brako \& Zarucchi la consideran como hierba siendo en realidad una planta leñosa del biotipo: Caméfitos leñosos postrados.

S. gamolepis forma cojines superiores a $1 \mathrm{~m}$ de diámetro libres o asociados a otras formas almohadilladas de la formación del césped de alta puna tales como Deyeuxia chrysophylla Phil., Austrocylindropuntia floccosa (Salm-Dyck) F. Ritter, Mniodes sp, Pycnophyllum spp

De Senecio canoi y S. sykorae Montesinos, morfológicamente similares a $S$. gamolepis (Montesinos et al, 2015), se diferencia en el color de la flor, mientras que las 2 primeras presentan la corola blanca, y no forman cojines mayores a $20 \mathrm{~cm}$; S. gamolepis la corola de sus- -flores son de color amarillo y forman cojines hasta de 1,40 m de diámetro.

Se reporta una nueva localidad de Senecio gamolepis Cabrera para los Altos Andes de la Cordillera de Vilcanota en la Región Cusco ampliando su distribución fitogeográfica.

\section{Agradecimientos}

Expreso mi agradecimiento al Proyecto GLORIA por invitarme a participar en calidad de Coordinador Responsable del Equipo Peruano en la expedición a la Cordillera de Vilcanota a mis recordados estudiantes y hoy colegas Jim Farfán, Marisol Jihuallanca, Amanda Delgado y Jesenia Vizcardo en el levantamiento de los sitios cimeros Pumachunta y Orqo Qocha en el año 2008, a Rosa Isela Meneses Directora del Herbario Nacional de La Paz-Bolivia y sus integrantes a Anton Seimon Traice Seimon, Stephan Halloy, Karina Yager por su participación y experiencia en la aplicación de la metodología validada Gloria.

A la Fundación para el desarrollo de la Ecología (FUN-ECO) por haber identificado a (IDSA ANTARQUI) para la ejecución del Estudio en la Cordillera de Vilcanota. A Perry Baker del Departamento de Geografía de la Universidad de Appalachian State de Carolina del Norte. USA por las expediciones sucesivas a la cordillera de Vilcanota y ser parte del equipo investigador.

Un especial agradecimiento a mí siempre estimado amigo y colega de trabajo de campo José Luis Venero Gonzales por compartir experiencias, dedicación al conocimiento científico y la revisión del manuscrito.

\section{Literatura consultada}

Beltrán H., A. Granda, B. León, A. Sagástegui, I. Sánchez \& M. Zapata. (2006). Asteráceas endémicas del Perú, en B. León, J. Roque, C. Ulloa Ulloa, N. Pitman, P.M. Jørgensen \& A. Cano (eds.), El Libro Rojo de las Plantas endémicas del Perú. Rev. Peru. Biol. Núm. Espec. 13:64s-164s. 
Beltran, H. (2016). Las Asteraceas (Compositae) del distrito de Laraos (Yauyos, Lima, Perú). Revista Peruana de Biología vol.23, num.2, pp 195-220. Univ. Nac. Mayor de San Marcos. Lima, Perú.

Brako, L. \& J. Zarucchi. (1993). Catálogo de las Angiospermas y Gimnospermas del Perú. Monographs in Systematic Botany from the Missouri Botanical Garden 45: 1-1286.

Cabrera, A. (1955). Notas sobre los Senecio sudamericanos VIII. Notas del Museo de la Plata. Botánica 18(89): 191-240.

Gentry, A. (1993). Reseña de la Flora Peruana XXiXXXXViii. En Brako \& Zarucchi, 1993

Gobierno Regional Cusco 2017, Propuesta de Área de Conservación Regional Ausangate. Gerencia Regional de Recursos Naturales y Gestión del Medio Ambiente.

Haral, P., M. Goptteried, D. Hohenwallner, K. Reitter \& G. Grabherr. (2003). Manual Para el trabajo de Campo del Proyecto GLORIA., Institute of Ecology and Conservation Biology University of Viena. Austria.pp 63.

IUCN. (2001). IUCN Red List Categ. Crit. v. 3.1 ii, 1-30. IUCN, Gland.

Komer, C. (1999). Alpine plant life fuctional plant ecology of high mountain ecosystems. Springer. Berlin, 338 pp.

León, B., J. Roque, C. Ulloa Ulloa, N. C.A.Pitman, P. M. Jørgensen \& A. Cano Echevarría. (2006) [2007]. En_El Libro Rojo de las Plantas Endémicas del Perú. Revista Peruana Biol. 13(núm. 2 especial): 1s-971s.

Montesinos, D., P. Gonzales, y E. Navarro. (2015). Senecio canoi (Compositae) Una especie nueva de los Andes del Perú. Anales del Jardín Botánico de Madrid 72(2): e026 2015. ISSN: 0211$\begin{array}{llll}13 & 2 & 2\end{array}$. d o i : http://dx.doi.org/10.3989/ajbm.2409.

Raunkiaer, C. (1934). The Life Forms of Plants and Statistical Plant Geografy, Oxford University Press.

Ulloa, C., J. Zarucchi. \& B. León. (2004). Diez años de Adiciones a la Flora del Perú 1993 - 2003. Arnaldoa. Ed. Especial, 7-242. 